\title{
Polypeptide profiles of whole cell lysate antigens of Trypanosoma Evansi isolated from the dogs by SDS-
}

\section{page}

\begin{abstract}
Trypanosoma evansi positive blood samples were collected from the dogs, multiplied in the wistar rats. Whole cell lysate antigen of Trypanosoma evansi parasites was prepared for further study by using the host cell free Trypanosoma evansi parasites. Study revealed a total of 13 polypeptide bands with relative molecular weight ranging from 25 to $82 \mathrm{kDa}$, when resolved in $10 \%$ sodium dodecyl sulphate polyacrylamide gel ectrophoresis and stained with Coomassie brilliant blue. Four major polypeptides of relative molecular weight of $33,36,41,43 \mathrm{kDa}$ and remaining nine polypeptides were minor appeared at molecular weight of $25,28,38,59,66,70,72,80,82 \mathrm{kDa}$. There is no variation in the antigenic profile of all the samples which were collected from the Rayalaseema region of Andhra Pradesh.
\end{abstract}

Keywords: Andhra pradesh, dogs, India, SDS-PAGE, Trypanosoma evansi
Volume 2 Issue 5 - 2015

\author{
Sivajothi S,' Rayulu VC,' Sudhakara Reddy B, ${ }^{2}$ \\ Bhaskar Reddy BV ${ }^{3}$ \\ 'Department of Veterinary Parasitology, Sri Venkateswara \\ Veterinary University, India \\ ${ }^{2}$ Department of Veterinary Medicine, Sri Venkateswara \\ Veterinary University, India \\ ${ }^{3}$ Senior scientist, Acharya NG Ranga Agricultural University, \\ India
}

Correspondence: Sivajothi S, College of Veterinary Science, Sri Venkateswara Veterinary University, Tiruapti, Andhra Pradesh, India, Email sivajothi579@gmail.com

Received: May II, 2015 | Published: August II, 2015

\section{Introduction}

Trypanosomosis caused by Trypanosoma evansi is one of the important vector borne diseases occurring in tropical and subtropical countries including in India. In India, T. Evansi infection is widely prevalent in different parts and is of significant economic importance in live stock production due to high degree of morbidity associated with decreased milk production, diminished working capacity and mortality of animals in fatal cases. Trypanosoma evansi can affect the buffaloes, cattle, camels, horses, dogs, tigers, sheep, goat, elephant, deer, cats and pigs. ${ }^{1,2}$ The disease is most severe in dogs and causes animal anemic, corneal opacity, tissue degenerative change and progressive emaciation. Common clinical signs in dogs include fever, weight loss, lethargy, signs of anemia, enlargement of the lymph nodes and dependent edema, jaundice, petechial hemorrhages of the mucous membranes, laryngeal edema and conjunctivitis. ${ }^{3,4}$

Diagnosis of trypanosomosis is based on clinical signs, demonstration of the parasites in the blood. Tests like slide ELISA, indirect fluorescent antibody test, colloidal dye immune binding technique, antibody-detection enzyme-linked immune sorbent assay, card agglutination test and polymerase chain reaction are useful for the diagnosis of infection. ${ }^{5-7}$ All the different tests vary with the antigenic variations associated with the Trypanosoma evansi. Severities in the development of the clinical signs vary with the antigenic variation in the organisms. Within the hosts, the chemical composition of outer layer the variable surface glycoprotein (VSG) of T. Evansi changes throughout the course of infection. ${ }^{8}$ The parasite has the ability to undergo such antigenic changes frequently and is known as antigenic variation. Worldwide different researchers observed the variations in the polypeptide profiles in different animals. ${ }^{9,10}$ But, there is no such type of studies on the isolates from the dogs. In this study we evaluated the antigenic diversity of the whole cell lysate (WCL) antigens of the different isolates collected from the different dogs in Rayalasema region of Andhra Pradesh, India.

\section{Materials and methods}

Whole blood was collected from the dogs (Figure 1) suspected for T. Evansi infection and processed for the diagnosis of T. Evansi by different diagnostic tests in the Rayalaseema region of Andhra Pradesh of India during the period 2009 to 2010. Wet blood film examination, peripheral blood smear examination, concentration methods and lab animal inoculation tests were carried out to confirm the disease (Figure 2). In the present study samples which were positive for T. Evansi parasites were used for preparation of whole cell lysate antigen. Whole blood was inoculated $(0.5 \mathrm{~mL})$ intra peritoneally into adult wistar rats for propagation. After five days of inoculation at high parasitemea blood was collected from the rats by cardiac puncture using heparin as anticoagulant. Trypanosomes were separated from the blood by anion-exchange chromatography using a Diethylaminoethyl (DEAE) cellulose column with phosphatesaline-glucose (PSG) solution, $\mathrm{pH}$ 8.0, as eluting buffer (Figure 3). Parasites eluted from the column were collected by centrifugation (a) $10,000 \mathrm{rpm}$ for 15 minutes, at $4^{\circ} \mathrm{C}$ and were washed 3times with PBS (pH 7.4). The final cell pellet was suspended in PBS ( $\mathrm{pH}$ 7.4) and was kept frozen at $-20^{\circ} \mathrm{C}$ until further use. Whole cell lysate (WCL) antigen was prepared from purified trypanosomes. The whole cell lysate antigen (WCL Ag) was partially purified after precipitating with 50percent saturated ammonium sulphate followed by extensive dialysis against PBS, $\mathrm{pH}$ 7.4. Protein concentration of the WCL Ag of $T$. evansi prepared in the present study was estimated and it was adjusted to $1.0 \mathrm{mg} / \mathrm{mL}$ in PBS, $\mathrm{pH} 8.0$ and stored at $-20^{\circ} \mathrm{C}$ in $1.0 \mathrm{~mL}$ aliquots. ${ }^{11}$

Polypeptide profile of WCL of T. Evansi was determined by SDS-PAGE as described by Laemmli. ${ }^{12}$ Briefly, the glass plates were properly cleaned and fixed by using spacers and clips. This assembled glass plates were placed in standing position. Five $\mathrm{ml}$ of $30 \%$ acrylamide, $2.3 \mathrm{ml}$ of sterile water, $2.5 \mathrm{ml}$ of $1.5 \mathrm{M}$ Tris $(\mathrm{pH} 8.8)$, $100 \mu 1$ of each $10 \%$ APS, and $10 \%$ SDS were added in a glass test 
tube. The glass plate's assembly was sealed with $1 \mathrm{ml}$ of the above solution along with $10 \mu 1$ of TEMED. After the sealing was done $10 \mu 1$ of TEMED was added to the $9 \mathrm{ml}$ of the remaining solution and poured in between the glass plates. Stocking gel solution $(3 \mathrm{ml})$ was prepared by mixing $2.1 \mathrm{ml}$ of water, $0.5 \mathrm{ml}$ of $30 \%$ acrylmide, $0.38 \mathrm{ml}$ of $1 \mathrm{M}$ Tris (pH 6.8), $30 \mu \mathrm{l}$ of each $10 \%$ SDS and $10 \%$ APS and $3 \mu 1$ of the TEMED and poured above the resolving gel, comb was placed and the gel was kept for $30 \mathrm{~min}$ for polymerization. Comb was removed and the wells were flushed with distilled water to remove unpolymerized acrylamide.

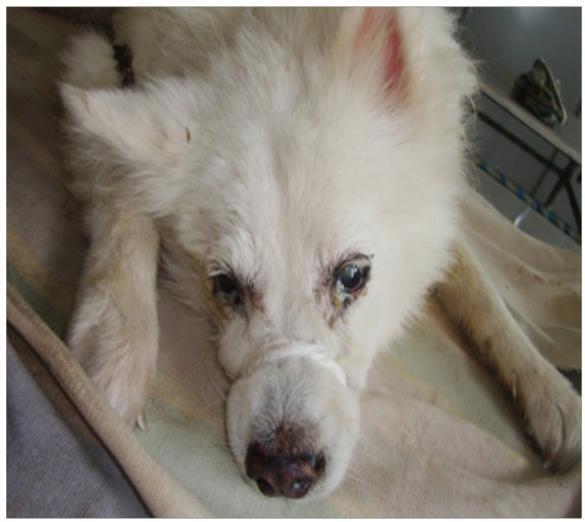

Figure I Dog affected with Trypanosoma evansi infection showing the corneal opacity and ocular discharges.

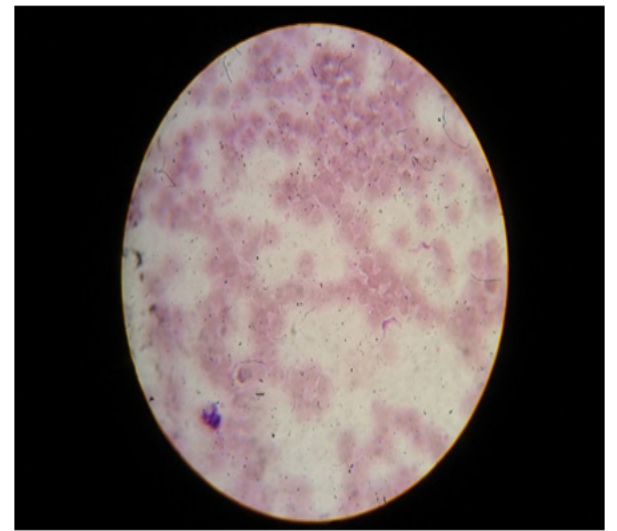

Figure 2 Presence of Trypanosoma evansi parasites in giemsa stained blood smear (I000X).

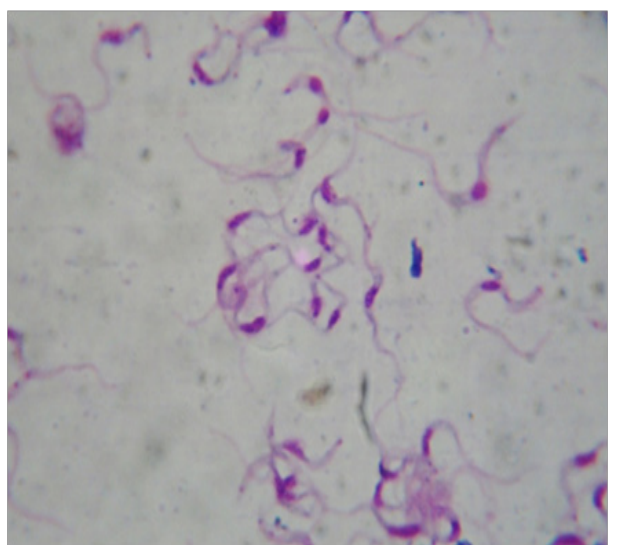

Figure 3 Leishman's stained host cell free Trypanosoma evansi organisms (I000X).
The gel mould was carefully disassembled after completion of electrophoresis and the gel was immersed into the staining trough containing Coomassie brilliant blue R-250 staining solution (CBBR) for $2 \mathrm{hrs}$. Later the gel was destained in destaining solution until the background became clear. After destaining the gel was transferred to storage solution. The markers used for electrophoresis were standard molecular weight markers (MWM) (Genei, Bangalore) with molecular weights ranging between $98 \mathrm{kDa}$ and $16 \mathrm{kDa}$. The distance migration from the origin to the middle of each protein band of samples and marker proteins were measured. A curve was drawn by tacking distance migration on $\mathrm{x}$-axis and molecular weights on $y$-axis for marker proteins on semi log graph paper. A straight line was drawn passing through maximum points. The molecular weight of the proteins was calculated by this standard curve.

\section{Results and discussion}

The polypeptide profiles of whole cell lysate antigens of $T$. Evansi which were prepared from the different dogs origins have been shown in Table $1 \&$ Figure 4 . In this total of 13 polypeptides were observed when resolved in $10 \%$ SDS-PAGE and stained with Commassie brilliant blue, with relative molecular weight ranging from 25 to 82 $\mathrm{kDa}$. Out of which four polypeptides i.e. $33,36,41$ and $43 \mathrm{kDa}$ were resolved as major polypeptides while the rest of $25,28,38,59,66$, $70,72,80,82$ were considered as minor polypeptides. In this study similar number of polypeptide bands were observed from whole cell lysate antigens of T. Evansi isolated from different dogs origins.

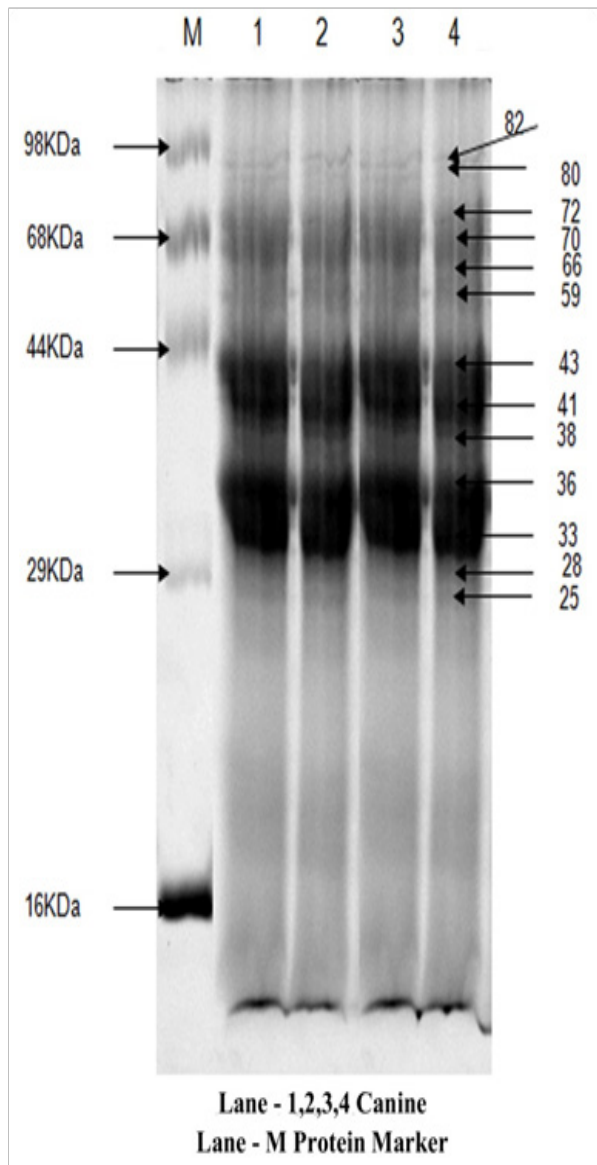

Figure 4 Polypeptide profiles of crude whole cell lysate antigens of $T$. Evansi isolated from dogs origin $(1,2,3,4)$ resolved on I0\% SDS-PAGE. 
Table I Approximate molecular mass $(\mathrm{kDa})$ of polypeptides in WCL antigen of Trypanosoma evansi isolated from the dogs

\begin{tabular}{ll}
\hline Polypeptide band no. & $\begin{array}{l}\text { Polypeptide size (kDa) in } \\
\text { T. Evansi whole cell lysate }\end{array}$ \\
\hline 1 & 25 \\
2 & 28 \\
3 & 33 \\
4 & 36 \\
5 & 38 \\
6 & 41 \\
7 & 43 \\
8 & 59 \\
9 & 66 \\
10 & 70 \\
II & 72 \\
12 & 80 \\
13 & 82 \\
Total no. of bands & 13 \\
\hline
\end{tabular}

Trypanosoma evansi has a huge range of hosts receptive and susceptible to the infection, in which it exhibits highly variable clinical effects, depending on the host and the geographical area. These characteristics make surra not only a multispecies but also a polymorphic disease. Data availability on the transmission dynamics of T. Evansi was very little. By assessing the biochemical, immunological, molecular and biological data of T. Evansi derived from distinct hosts and geographical regions can be help full or the assessment of the severity. Very limited antigenic diversity has been described among T. Evansi isolates. This characteristic reinforces the importance of the host's humoral immune response and suggests the possibility of controlling this enzootic disease through immunoprophylactic methods. ${ }^{13}$ Whole cell lysate (WCL) antigen of T. Evansi isolate was prepared following the method of Sivajothi et al. ${ }^{14}$ The approximate molecular weights of these polypeptides were ranged from 25 to $82 \mathrm{kDa} .{ }^{14}$ Hence, the present findings were compared with the polypeptide profiles of the cattle isolates of $T$. Evansi which revealed the presence of additional bands indicating the variations in the isolates between the different hosts. Using the same method of preparation of antigen, Giardina et al., ${ }^{15}$ observed 25 polypeptides of molecular weights between 97 and $14.8 \mathrm{kDa}$, but they did not do study on T. Evansi which were collected from different hosts. ${ }^{15}$

In the present study similarity in polypeptide profiles were observed which might be due to non significant antigenic diversity between stocks of T. Evansi collected from the dogs. This might be due to parasite factors, which include the amino acid sequence on the surface of the parasite, which is reported to be influenced the exposure as well as physical differences in between different parasite environment. This might affect the release of the soluble form of variable surface glycoprotein (VSG) as also expressed by various workers. ${ }^{16}$ No variations in the antigenic characterization in the present study may be due to change in VSG of T. Evansi is not a constant phenomenon in same type of host. It is relative, depending on some intrinsic factors related to the physiological environment of host and parasite. But, this is the first report on polypeptides of dog isolates. Laha et al. ${ }^{10}$ reported that the polypeptide bands of WCL Ags of T. Evansi isolated from buffalo, horse and cattle origins were similar and observed seven major polypeptides in the ranges of 86-87, 74-75, 61-62, 51-53, 39, $34-35$ and $13 \mathrm{kDa}$ and four minor polypeptides in the ranges of 93-95, 46-47, 28-29 and 25-26kDa. ${ }^{10}$ Using crude sonicated antigens of $T$. Evansi of cattle and camel isolates of northern India, Pareek et al., ${ }^{17}$ observed eight polypeptide bands common in both isolates in $12 \%$ SDS-PAGE. ${ }^{17}$

Salivarian trypanosomes are capable of change their surface coat during the course of infection. ${ }^{18}$ The African trypanosomes variant surface coat (VSG) is reported to be a single $10-15 \mathrm{~nm}$ thick coat of glycoproteins with a MW ranging between 53 and $63 \mathrm{kDa} .{ }^{19}$ In this study all the isolates from the dogs also had molecular weight $59 \mathrm{kDa}$ (minor polypeptides). Previously, we described the homogeneity of T. Evansi isolates derived from cattle in polypeptide profiles of WCL antigen characterization. In spite of this homogeneity we observed differences in the virulence pattern of the T. Evansi isolates among the different species of the animals. Changes in the polypeptide profiles of WCL antigens of T. Evansi can causes variations in the immune responses in different hosts.

In the present study there is no antigenic variations were recorded between the T. Evansi isolates from different dogs. But, these findings were differed from the T. Evansi isolates samples collected from the cattle in the same region. Hence, studies are required to record the antigenic variations due to different propagation methods of T. Evansi isolates.

\section{Conclusion}

Present study revealed a total of 13 polypeptide bands with relative molecular weight ranging from 25 to $82 \mathrm{kDa}$, when resolved in $10 \%$ sodium dodecyl sulphate polyacrylamide gel electrophoresis and stained with Coomassie brilliant blue. There is no variation in the antigenic profile of all the dog samples which were collected from the Rayalaseema region of Andhra Pradesh.

\section{Acknowledgements}

None.

\section{Conflict of interest}

Author declares that there is no conflict of interest.

\section{References}

1. Sivajothi S, Rayulu VC, Reddy BS. Haematological and biochemical changes in experimental Trypanosoma evansi infection in rabbits. $J$ Parasit Dis. 2015;39(2):216-220.

2. Dargantes AP, Mercado RT, Dobson RJ, et al. Estimating the impact of Trypanosoma evansi infection (surra) on buffalo population dynamics in southern Philippines using data from cross-sectional surveys. Int $J$ Parasitol. 2009;39(10):1109-1114

3. Reddy BS, Kumari KN, Sivajothi S, et al. Haemato-biochemical and thyroxin status in Trypanosoma evansi infected dogs. $J$ Parasit Dis. 2014.

4. Herrera HM, Aquino LP, Menezes RF, et al. Trypanosoma evansi experimental infection in the South American coati (Nasua nasua): clinical, parasitological and humoral immune response. Vet Parasitol. 2001;102(3):209-216 
5. OIE. Trypanosoma evansi infections (including surra). In: OIE Terrestrial Manual 2008. Office International des Epizooties World Health Organization for Animal Health. Paris; 2008. p. 352-360.

6. Sivajothi S, Rayulu VC, Reddy BS. Development of Slide Enzyme Linked Immunosorbent Assay (SELISA) for Detection of Trypanosoma evansi Infection in Bovines. Journal of Advanced Veterinary Research. 2012;2(1):15-17

7. Sivajothi S, Rayulu VC, Malakondaiah P, et al. Colloidal Dye Immunobinding Assay for Detection of Trypanosoma evansi Antibodies in Animals. Int J Livest Res. 2013;3(3):48-56.

8. Zheng RJ, Sheng J, Qui QP, et al. Comparison of protein components and antigenicities of various strains of Trypanosoma evansi from Zhejiasg, Yunan and Anhui provinces. Chinese J Vet Sci Technol. 1990;2:7-9.

9. Queiroz AO, Legey AP, Xavier SCC, et al. Specific antibody levels and antigenic recognition of wistar rats inoculated with distinct isolates of Trypanosoma evansi. Mem Inst Oswaldo Cruz. 2001;96(7):965-972.

10. Laha R, Sasmal NK, Bandyopadhyay S. Comparative polypeptide profiles of whole cell lysate antigens of Trypanosoma evansi isolated from three different hosts of eastern India. J Protozool Res. 2008;18:11-16.

11. Sivajothi S, Rayulu VC, Malakondaiah P, et al. Detection of antibodies against Trypanosoma evansi in Sheep by Indirect ELISA in Rayalaseema region of Andhra Pradesh. Journal of Advanced Veterinary Research. 2014;4(3):88-92.
12. Laemmli UK. Cleavage of structural proteins during the assembly of the head of bacteriophage T4. Nature. 1970;227(5259):680-685.

13. Jones TW, McKinnell CD. Antigenic variation in Trypanosoma evansi: a comparison of the predominant variable antigen type repertoires of stocks from the Sudan. Trop Med Parasitol. 1985;36(4):205-209.

14. Sivajothi S, Rayulu VC, Reddy BVB, et al. Polypeptide profiles of South Indian isolate of Trypanosoma evansi. Journal of Parasitic Diseases. 2014.

15. Giardina S, Paganico G, Urbani G, et al. A Biochemical and Immunological Comparative Study on Trypanosoma equiperdum and Trypanosoma evansi. Vet Res Commun. 2003;27(4):289-300.

16. Uche E, Ross CA, Jones TW. Identification of the surface components of Trypanosoma evansi. Res Vet Sci. 1992;53(2):252-253.

17. Pareek RK, Pathak KML, Kumar S, et al. Polypeptide profile of Trypanosoma evansi isolates of camel and cattle origin. $J$ Vet Parasitol. 1999;13:53-54.

18. Overath P, Chaudhri M, Steverding D, et al. Invariant surface proteins in bloodstream form of Trypanosoma brucei. Parasitol Today. 1994;10(2):53-58.

19. Richards FF. The surface of African trypanosomes. J Protozool. 1984;31(1):60-64. 